\title{
Artificial Intelligence for Pediatric Ophthalmology
}

\author{
Julia E. Reid, $M D^{\ddagger, \dagger}$ and Eric Eaton, $P h D^{\ddagger}$
}

\begin{abstract}
Purpose of review
Despite the impressive results of recent artificial intelligence $(\mathrm{Al})$ applications to general ophthalmology, comparatively less progress has been made toward solving problems in pediatric ophthalmology using similar techniques. This article discusses the unique needs of pediatric ophthalmology patients and how Al techniques can address these challenges, surveys recent applications of Al to pediatric ophthalmology, and discusses future directions in the field.
\end{abstract}

\section{Recent findings}

The most significant advances involve the automated detection of retinopathy of prematurity (ROP), yielding results that rival experts. Machine learning (ML) has also been successfully applied to the classification of pediatric cataracts, prediction of post-operative complications following cataract surgery, detection of strabismus and refractive error, prediction of future high myopia, and diagnosis of reading disability via eye tracking. In addition, ML techniques have been used for the study of visual development, vessel segmentation in pediatric fundus images, and ophthalmic image synthesis.

\begin{abstract}
Summary
Al applications could significantly benefit clinical care for pediatric ophthalmology patients by optimizing disease detection and grading, broadening access to care, furthering scientific discovery, and improving clinical efficiency. These methods need to match or surpass physician performance in clinical trials before deployment with patients. Due to widespread use of closed-access data sets and software implementations, it is difficult to directly compare the performance of these approaches, and reproducibility is poor. Open-access data sets and software implementations could alleviate these issues, and encourage further Al applications to pediatric ophthalmology.
\end{abstract}

Keywords

pediatric ophthalmology, machine learning, artificial intelligence, deep learning

\section{INTRODUCTION}

The increased availability of ophthalmic data, coupled with advances in artificial intelligence (AI) and machine learning (ML), offer the potential to positively transform clinical practice. Recent applications of ML techniques to general ophthalmology have demonstrated the potential for automated disease diagnosis [1, automated prescreening of primary care patients for specialist referral [2], and scientific discovery 3], among others. Acting as a complement to ophthalmologists, these and future applications have the potential to optimize patient care, reduce costs and barriers to access, limit unnecessary referrals, permit objective monitoring, and enable early disease detection.

To date, most AI applications have focused on adult ophthalmic diseases, as discussed by several reviews 4 11. Comparatively little progress has been made in applying AI and ML techniques to pedi- atric ophthalmology, despite the pressing need. In the United States, there is a shortage of pediatric ophthalmologists 12 and fellowship positions continue to go unfilled 13. Globally, this shortage is even more pronounced and devastating - for example, retinopathy of prematurity (ROP), now in its third epidemic, has resulted in irreversible blindness in over 50,000 premature infants due to worldwide shortages of trained specialists and other barriers to adequate care 14, 15.

\footnotetext{
Nemours / Alfred I. duPont Hospital for Children, Division of Pediatric Ophthalmology, Wilmington, DE; †Thomas Jefferson University, Departments of Pediatrics and Ophthalmology, Philadelphia, PA; and $\ddagger$ University of Pennsylvania, Department of Computer and Information Science, Philadelphia, PA
}

Correspondence to Julia E. Reid, MD, Division of Pediatric Ophthalmology, 1600 Rockland Road, Wilmington, DE 19803, USA. email: julia.e.reid@nemours.org 


\section{KEY POINTS}

- Pediatric ophthalmology has unique aspects that must be considered when designing $\mathrm{Al}$ applications, including disease prevalence, cause, presentation, diagnosis, and treatment, which differ from adults.

- Most recent Al applications focus on ROP or congenital cataracts, although many other areas of pediatric ophthalmology could benefit from Al.

- Reproducibility and comparability between current Al approaches is poor, and would be improved with open-access data sets and software implementations.

- Evaluation on experimental data sets should be augmented with clinical validation prior to deployment with patients.

\section{UNIQUE CONSIDERATIONS FOR PEDIATRIC OPHTHALMOLOGY}

Ophthalmic disease prevalence, cause, presentation, diagnosis, and treatment all differ between adult and pediatric patients - dissimilarities that are important to consider when developing AI applications.

Common diseases in children include amblyopia, strabismus, nasolacrimal duct obstruction (NLDO), retinopathy of prematurity (ROP), and congenital eye diseases. The adult population, by contrast, is affected by cataracts, dry eye, macular degeneration, diabetic retinopathy, and glaucoma. For diseases that occur in both children and adults, the presentation, cause, and treatment often differ. Glaucoma is a good example, as the cause and presentation in congenital glaucoma patients are both unlike those in adultonset glaucoma patients. Optimal management of glaucoma, including surgery, also differs for these two populations.

Infants and children have distinct characteristics from adults that affect their ophthalmology visits. Given their developmental capabilities, there is generally less information gleaned from a single eye exam of a child, so several visits may be required to accurately diagnose or characterize that child's disease. There is also a stronger reliance on the objective exam because of the infant's or child's inability to effectively communicate. Children's short attention spans and unpredictable behavior often necessitate a quick exam that allows the physician to gain the child's trust while keeping him or her at ease. Despite this, there are portions of the clinic visit that take longer, such as restraining a child to administer dilating drops and then waiting for that child to be fully cyclopleged. Ancillary testing that requires patient cooperation may not be possible in an awake child, and eye exams under anesthesia are not uncommon. Similarly, children are typically placed under general anesthesia for eye procedures, whereas adults may require only topical or local anesthesia. Techniques for more accurate diagnosis and disease prediction could help reduce the high cost and risk of repeated exams and surgeries under anesthesia.

Other distinguishing factors pertain to the pediatric patient's growth and development. In most children, visual development occurs from birth until age 7 or 8; eye diseases affecting children during this period can cause permanent vision loss due to amblyopia or reduced visual abilities. Additionally, during development, significant ocular growth occurs, causing changes in refractive error that complicate surgical planning for congenital cataract patients.

Retinal imaging, too, differs for pediatric and adult patients. Factors such as children's lack of fixation and small pupils can create blur, partial occlusion, and illumination defects, all of which degrade image quality. For infants being screened for ROP, their fundus images are more variable and have more visible choroidal vessels, making classification comparatively difficult 16 .

\section{CLINICAL APPLICATIONS OF AI}

This section surveys recent AI applications to pediatric ophthalmology, organized by disease (see Table 1). The approaches discussed in this survey would more precisely be called applications of MLthe largest subfield of AI concerned with learning models from data. We have provided a brief overview of AI and ML and their relationship in supplemental material, but the interested reader is encouraged to consult a more extensive tutorial on these topics [e.g. 5]. To limit its scope, this review focuses on applications with a goal of having the AI aspects directly impact clinical practice; we omit studies where ML was used primarily for statistical analysis.

\section{Retinopathy of Prematurity (ROP)}

The most significant AI advances in pediatric ophthalmology apply to ROP, a leading cause of childhood blindness worldwide [14, 15, 40]. In addition to the shortage of trained providers [14, 15, 41], ROP exams are difficult, clinical impressions are subjective and vary among examiners [23, 42, 43], and disease management is time-intensive, requiring several serial exams. AI applications have focused on detecting the presence and grading of ROP or plus disease from 
Table 1. Summary of ML-based techniques for pediatric ophthalmic disease detection and diagnosis

\begin{tabular}{|c|c|c|c|c|c|c|}
\hline $\begin{array}{l}\text { Approach } \\
\text { (Approx. devel. year) }\end{array}$ & Predicted category & $\begin{array}{l}\text { Sensitivity } \\
(\%)\end{array}$ & $\begin{array}{l}\text { Specificity } \\
(\%)\end{array}$ & AUROC & $\begin{array}{l}\text { Accuracy } \\
(\%)\end{array}$ & Method summary \\
\hline \multicolumn{7}{|c|}{ Retinopathy of prematurity (ROP) } \\
\hline $\begin{array}{l}\text { DeepROP 17"- } \\
(2018)\end{array}$ & $\begin{array}{l}\text { Experimental data set } \\
\text { Presence of ROP } \\
\text { Severe (vs Mild) ROP } \\
\text { Clinical test } \\
\text { Presence of ROP } \\
\text { Severe (vs Mild) ROP }\end{array}$ & $\begin{array}{l}96.64 \\
88.46\end{array}$ & $\begin{array}{l}99.33 \\
92.31\end{array}$ & $\begin{array}{l}0.995 \\
0.951\end{array}$ & $\begin{array}{l}97.99 \\
90.38 \\
95.55 \\
76.42\end{array}$ & $\begin{array}{l}\text { Cloud-based platform. Set of } \\
\text { fundus images } \rightarrow \text { two CNNs } \\
\text { (modified Inception-BN nets } \\
\text { pretrained on ImageNet): one } \\
\text { predicts presence, and the } \\
\text { other severity }\end{array}$ \\
\hline $\begin{array}{l}\text { i-ROP-DL 18"- } \\
(2018)\end{array}$ & $\begin{array}{l}\text { Clinically significant ROP } \\
\text { Type } 1 \text { ROP } \\
\text { Type } 2 \text { ROP } \\
\text { Pre-plus disease }\end{array}$ & $\begin{array}{r}- \\
94 \\
- \\
-\end{array}$ & $\begin{array}{r}- \\
79 \\
- \\
-\end{array}$ & $\begin{array}{l}0.914 \\
0.960 \\
0.867 \\
0.910\end{array}$ & $\begin{array}{l}- \\
- \\
- \\
-\end{array}$ & $\begin{array}{l}\text { Applies a linear formula to } \\
\text { the probabilities output by } \\
\text { i-ROP-DL (see below) to yield } \\
\text { a severity score on a } 1-9 \text { scale }\end{array}$ \\
\hline $\begin{array}{l}\text { MiGraph 19] } \\
\text { (2016) }\end{array}$ & Presence of ROP & 99.4 & 95.0 & 0.98 & 97.5 & $\begin{array}{l}\text { SIFT features from image } \\
\text { patches } \rightarrow \text { multiple instance } \\
\text { learning graph-kernel SVM }\end{array}$ \\
\hline $\begin{array}{l}\text { VesselMap 20 } \\
\text { (2007) }\end{array}$ & $\begin{array}{l}\text { Severe ROP } \\
\text { From mean arteriole diameter } \\
\text { From mean venule diameter }\end{array}$ & - & - & $\begin{array}{l}0.93 \\
0.87\end{array}$ & $\begin{array}{l}- \\
-\end{array}$ & $\begin{array}{l}\text { Semiautomated tool that uses } \\
\text { classic image analysis to mea- } \\
\text { sure vessel diameter }\end{array}$ \\
\hline \multicolumn{7}{|c|}{ ROP: Plus or pre-plus disease } \\
\hline $\begin{array}{l}\text { i-ROP-DL 21'] } \\
(2018)\end{array}$ & $\begin{array}{l}\text { Plus disease } 18^{-\cdot]} \\
\left.\text { Pre-plus disease } 18^{m-}\right] \\
\text { Plus disease }\left[21^{-}\right] \\
\text {Pre-plus or worse disease }\left[21^{-}\right]\end{array}$ & $\begin{array}{l}- \\
- \\
93 \\
100\end{array}$ & $\begin{array}{r}- \\
- \\
94 \\
94\end{array}$ & $\begin{array}{l}0.989 \\
0.910 \\
0.98 \\
0.94\end{array}$ & $\begin{array}{c}- \\
- \\
91.0 \\
-\end{array}$ & $\begin{array}{l}\text { CNN-output (U-net) ves- } \\
\text { sel segmentations } \rightarrow \text { CNN } \\
\text { (InceptionV1 pretrained on } \\
\text { ImageNet) to classify as } \\
\text { normal/pre-plus/plus }\end{array}$ \\
\hline $\begin{array}{l}\text { CNN + Bayes 16] } \\
(2016)\end{array}$ & $\begin{array}{r}\text { Plus disease }(\text { per image) } \\
\text { (per exam) }\end{array}$ & $\begin{array}{l}82.5 \\
95.4\end{array}$ & $\begin{array}{l}98.3 \\
94.7\end{array}$ & - & $\begin{array}{l}91.8 \\
93.6\end{array}$ & $\begin{array}{l}\text { CNN (InceptionV1 pretrained } \\
\text { on ImageNet) adapted to out- } \\
\text { put the Bayesian posterior }\end{array}$ \\
\hline $\begin{array}{l}\text { i-ROP 22] } \\
(2015)\end{array}$ & $\begin{array}{l}\text { Plus disease } \\
\text { Pre-plus or worse disease }\end{array}$ & $\begin{array}{l}93 \\
97\end{array}$ & - & - & $\begin{array}{r}95 \\
-\end{array}$ & $\begin{array}{l}\text { SVM with a kernel derived } \\
\text { from a GMM of tortuosity and } \\
\text { dilation features from manually } \\
\text { segmented images }\end{array}$ \\
\hline $\begin{array}{l}\text { Naïve Bayes 23] } \\
\text { (2015) }\end{array}$ & $\begin{array}{l}\text { Plus/pre-plus/none (SVM-RFE) } \\
\text { Plus disease (ReliefF) }\end{array}$ & - & - & $\begin{array}{l}- \\
-\end{array}$ & $\begin{array}{l}79.41 \\
88.24\end{array}$ & $\begin{array}{l}\text { Naïve Bayes with SVM-RFE or } \\
\text { ReliefF vessel feature selection }\end{array}$ \\
\hline $\begin{array}{l}\text { CAIAR 24] } \\
(2008)\end{array}$ & $\begin{array}{l}\text { Plus (from venule width) } \\
\text { Plus (from arteriole tortuosity) }\end{array}$ & $\begin{array}{l}- \\
-\end{array}$ & - & $\begin{array}{l}0.909 \\
0.920\end{array}$ & $\begin{array}{l}- \\
-\end{array}$ & $\begin{array}{l}\text { Generative vessel model fit to } \\
\text { a multi-scale representation of } \\
\text { the retinal image }\end{array}$ \\
\hline $\begin{array}{l}\text { ROPtool 26] } \\
\text { (2007) }\end{array}$ & $\begin{array}{l}\text { Plus tortuosity (eye) } \\
\text { (quadrant) } \\
\text { Pre-plus tortuosity (quadrant) }\end{array}$ & $\begin{array}{l}95 \\
85 \\
89\end{array}$ & $\begin{array}{l}78 \\
77 \\
82\end{array}$ & $\begin{array}{l}- \\
0.885 \\
0.875\end{array}$ & $\begin{array}{c}87.50 \\
80.63 \\
-\end{array}$ & $\begin{array}{l}\text { User-guided tool that traces } \\
\text { centerlines of retinal vessels to } \\
\text { measure tortuosity }\end{array}$ \\
\hline $\begin{array}{l}\text { RISA 27] } \\
(2005)\end{array}$ & $\begin{array}{l}\text { Plus disease (from arteriole } \\
\text { and venule curvature and } \\
\text { tortuosity, venule diameter) }\end{array}$ & 93.8 & 93.8 & 0.967 & - & $\begin{array}{l}\text { Logistic regression on geomet- } \\
\text { ric features computed for each } \\
\text { segment of the vascular tree }\end{array}$ \\
\hline $\begin{array}{l}\text { IVAN 24] } \\
(2002)\end{array}$ & Plus (from venule width) & - & - & 0.909 & - & $\begin{array}{l}\text { Measures vessel width via clas- } \\
\text { sic image analysis }\end{array}$ \\
\hline
\end{tabular}

Abbreviations: AUROC - area under the receiver operating characteristic curve; GMM - Gaussian mixture model

digital fundus photos. Beyond the benefits of automated ROP screening and objective assessment, digital retinal imaging may cause less pain and stress for infants undergoing ROP screening compared to indirect ophthalmoscopy 44 and enable neonatology-led screening programs 45 .

Early computational approaches to detecting plus disease from fundus images focused on vessel tortuosity. One early attempt to objectively quantify tortuosity used the spatial frequency of manual vessel tracings 46 . Since then, there have been several tools developed to determine vessel tortuosity and width via classic image analysis, including Vessel Finder 47, VesselMap [20, ROPtool 26], RISA 27. 48, 49, CAIAR 24, 25], and IVAN 24, 50, all of which require at least one manual step from the user. Recent work suggests other potential vessel measurements correlated with plus disease, such as a decrease in the openness of the major temporal arcade angle [51. Once extracted, retinal vessel measurements have been used as features for various predictive models of plus disease, including linear models such as logistic regression 27 and naïve Bayes 23], as well as non-linear models trained by support vector machines 
Table 1. (Continued)

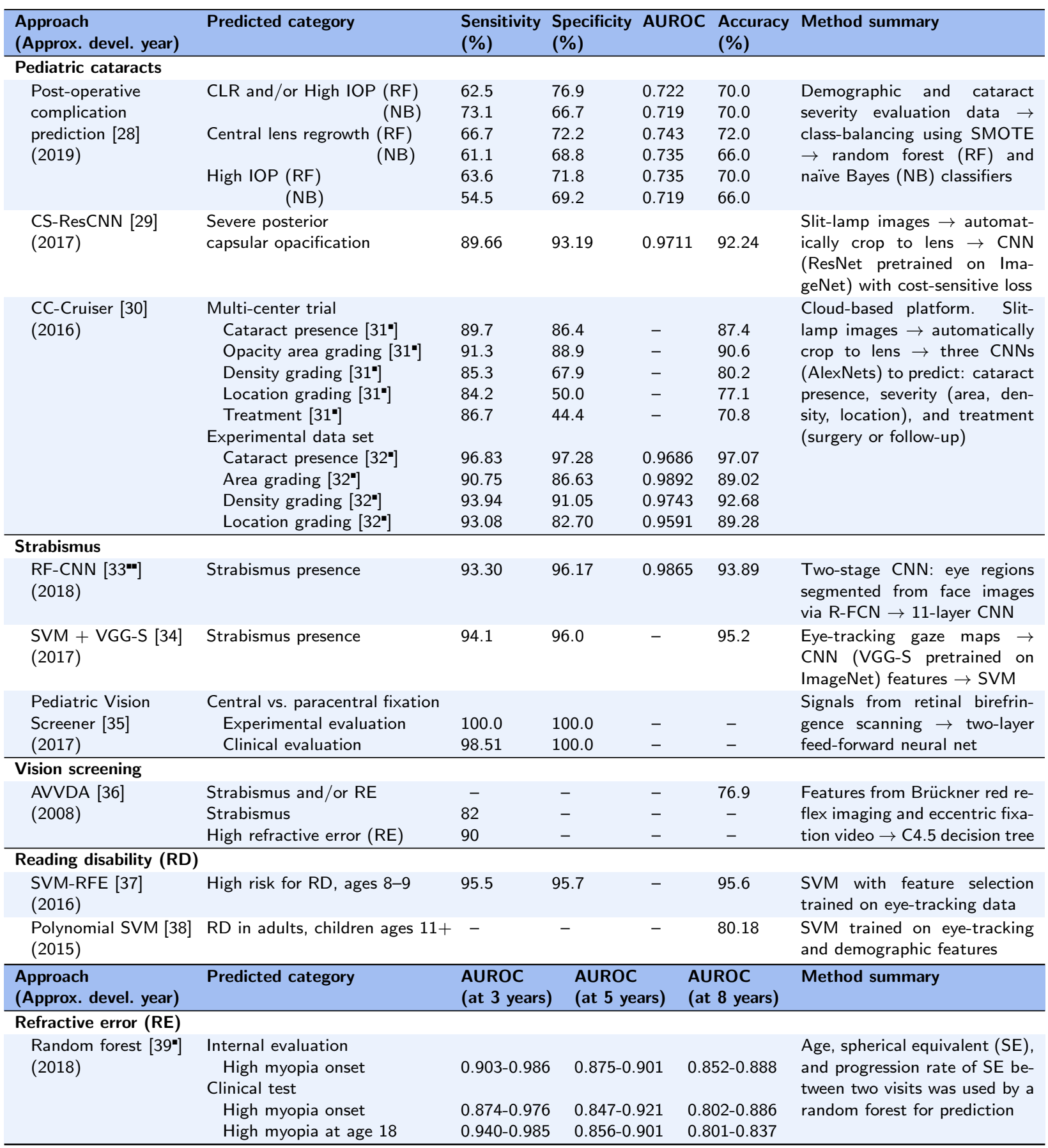

(SVMs) 22]. For predicting ROP, Rani et al. [19] also employ an SVM, but instead use SIFT 52 features extracted from retinal image patches and frame the problem in a multiple instance learning [53] setting.

Recent approaches to ROP and plus disease detection are mostly based on convolutional neural networks $(\mathrm{CNN})$, which take fundus images as input and do not require manual annotation. These sys- tems, which include Worrall et al. 16], i-ROP-DL 18' 21", and DeepROP [17-], demonstrate agreement with expert opinion $16,18^{-}$and better disease detection than some experts $\left[17^{-\prime}, 21^{-1}\right.$.

Like many ML methods, these systems can provide a confidence score in their predictions. i-ROPDL exploits this notion directly by combining the prediction probabilities via a linear formula to compute 
Table 2. Pediatric ROP data sets used in deep learning

\begin{tabular}{|c|c|c|c|c|}
\hline Approach & Data set & Patients & Images & Labels \\
\hline $\begin{array}{l}\text { DeepROP } \\
{\left[17^{-"}\right]}\end{array}$ & Chengdu & 1,273 & 20,795 & $\begin{array}{l}\text { normal, mild ROP, } \\
\text { severe ROP }\end{array}$ \\
\hline $\begin{array}{l}\text { i-ROP-DL } \\
\left.21^{*}\right]\end{array}$ & i-ROP & 898 & 5,511 & $\begin{array}{l}\text { normal, plus, } \\
\text { pre-plus }\end{array}$ \\
\hline CNN + Bayes & Canada & 35 & $\begin{array}{r}1,459 \\
106\end{array}$ & normal, plus \\
\hline
\end{tabular}

an ROP severity score, which can serve as an objective quantification of disease; a similar idea could provide finer grading of plus disease [21].

For their core predictive networks, all these CNNbased systems use versions of the Inception architecture 54, 55] with transfer learning [56, 57] by pretraining on ImageNet, giving them similar foundations. However, these approaches differ in preprocessing (e.g., i-ROP-DL 21" uses a U-net [58 to perform automatic vessel segmentation) and postprocessing (e.g., i-ROP-DL $18^{--}$outputs the ROP severity score; Worrall et al. [16] outputs the Bayesian posterior). DeepROP processes a set of fundus images per case, taking a multiple instance learning $[53$ approach, while the other two deep learning methods classify single images. The other key difference is that these systems are trained on different non-public ROP data sets of varying sizes and labelings (Table 2). The use of non-public data sets and closed implementations (only DeepROP is open source) complicates comparison and reproducibility [59].

Current methods for ROP detection are capable of coarse-grained classification, such as discriminating severe from mild ROP; they do not specifically assess disease stage or zone (e.g., $17^{\mathbf{*}}$ ). In fact, all systems except DeepROP 17" and MiGraph [19 examine only the posterior pole view, either ignoring other views or explicitly cropping them out. While the literature suggests that severe disease rarely develops without changes in posterior pole vasculature [60], providing additional outputs of the zone and stage could improve the interpretability of the system's assessment and improve performance.

\section{Pediatric Cataracts}

Pediatric cataracts are more variable than adult cataracts, and surgical removal depends upon cataract severity and deprivational amblyopia risk. Slit lamp exams enable cataract visualization but can be challenging and subjective, and slit lamp image quality can vary (e.g., based on the child's cooperativeness, image amplification, and interference from eyelashes and other eye disease or structures) [32].
CC-Cruiser 30 32 is a cloud-based platform that can automatically detect cataracts from slitlamp images, grade them, and recommend treatment. After automatically cropping the slit-lamp image to the lens region, it uses three separate CNNs (modified AlexNets [61]) to predict three aspects: cataract presence, grading (opacity area, density, location), and treatment recommendation (surgery or non-surgical follow-up). CC-Cruiser was evaluated in a multicenter randomized controlled trial within five ophthalmology clinics, demonstrating significantly lower performance in diagnosing cataracts $(87.4 \%)$ and recommending treatment $(70.8 \%)$ than experts $(99.1 \%$ and $96.7 \%$, respectively), but achieving high patient satisfaction for its rapid evaluation $31^{-}$.

Children who require surgery face potential complications that differ from those that adults face 62 . Zhang et al. applied random forests and naïve Bayes classifiers to predict two common post-operative complications, central lens regrowth and high intraocular pressure (IOP), from a patient's demographic information and cataract severity evaluation 28 . Another approach [29] uses a CNN to detect severe posterior capsular opacification warranting surgery, employing a ResNet 63 pretrained on ImageNet with a costsensitive loss to handle data set imbalance.

\section{Strabismus}

Strabismus affects 1 in 50 children and can cause amblyopia, interfere with binocularity, and have lasting psychosocial effects 64 68. A CNN was used to detect strabismus based on visual manifestation in the eye regions of facial photos $33^{-*}$, which would be especially useful for telemedical evaluation. For inoffice evaluation, which in contrast permits the use of specialized screening instruments, strabismus can be detected using a CNN based on fixation deviations from eye-tracking data 34], or with very high sensitivity and specificity from retinal birefringence scanning [35].

\section{Vision Screening}

Like strabismus, refractive error can cause amblyopia, but is difficult for pediatricians to detect. Instrument-based vision screening is recommended 69 and most devices have adjustable thresholds for signaling a screening failure. Using video frames from one such instrument that combines Brückner pupil red reflex imaging and eccentric photorefraction, Van Eenwyk et al. trained a variety of ML classifiers to detect amblyogenic risk factors in young children, with the most successful being a C4.5 decision tree [70]. 


\section{Reading Disability}

Reading disability affects approximately $10 \%$ of children 38 , but objective and efficient testing for it is lacking 37. Abnormal eye tracking is non-causally associated with reading disability 37,38 . Two studies used SVMs to identify reading disability from eye movements during reading, either predicting reading disability risk in children ages 8-9 [37], or detecting reading disability in adults and children ages $11+$ 38]. The children in both of these studies are older than the optimal age for diagnosis, so validation in a younger cohort could be useful.

\section{Refractive Error}

High myopia is associated with numerous visionthreatening complications 71 . Children at risk for high myopia can take low-dose atropine to halt or slow myopic progression 72,73 , but it can be difficult to determine for which children to recommend this treatment $39^{-}$. Lin et al. 39" predicted high myopia in children from clinical measures using a random forest, showing good predictive performance for up to 8 years into the future. Further work has the potential to guide prophylactic treatment.

\section{Non-Pediatric Applications}

AI has been applied to various adult ophthalmic diseases, including diabetic retinopathy [1, 74 77], AMD 78 83, sight-threatening retinal disease $2,84,89$, glaucoma 90 92, intraocular lens calculation 93], and keratoconus 94. It has also been used for robotassisted repair of epiretinal membranes [95], retinal vessel segmentation 96 99, and systemic disease prediction from fundus images 100]. For a detailed review, see [4 11].

\section{OTHER OPHTHALMIC APPLICATIONS}

This section reviews applications of ML to pediatric ophthalmology that are not tied to specific diagnoses.

\section{Visual Development}

ML has the potential to provide scientific insight into visual development. For example, adults who had cataract surgery and aphakic correction in infancy have exhibited diminished facial processing capabilities 101, 102. This impairment was originally blamed on early visual deprivation [101, 102, but more recently, it was conjectured to be caused by the aphakic correction and high initial acuity experienced by these infants $103^{-1}$. The hypothesis is that many visual proficiencies, such as facial recognition, are facilitated by the gradual increase in visual acuity during normal visual development. When tested in CNNs via initial training with blurred images, gradual acuity development increased generalization performance and encouraged the development of receptive fields with a broader spatial extent 103"These results provide a possible explanation for the decreased visual proficiencies of congenital cataract patients, and suggest the potential for temporary refractive undercorrection to help restore visual development 103".

\section{Pediatric Retinal Vessel Segmentation}

Although many programs have been developed for vessel segmentation in adults or premature infants, fundus images in older children have unique traits, including light artifacts, that complicate segmentation 104. Fraz et al. 104 developed an ensemble of bagged decision trees that use multi-scale analysis with multiple filter types to do vessel segmentation in pediatric fundus images. Another tool, CAIAR 25, has been validated in school-aged children $[105]$. $\overrightarrow{\mathrm{CA}}-$ IAR was first applied to infants with ROP and uses a generative model of the vessels fit via maximum likelihood to a multi-scale representation of the retinal image 25.

\section{Ophthalmic Image Synthesis}

Through their multi-layered representation, deep learning methods such as generative adversarial networks 106 are able to synthesize novel realistic images, including retinal fundus images 107, 108. Such synthesized images can compensate for data scarcity, preserve patient privacy, and depict variations on or combinations of diseases for resident education 109, 110.

One recent technique to synthesize highresolution images, progressive growing of GANs (PGGANs), was used to synthesize realistic fundus images of ROP (see examples in Figure 1) 111. The PGGAN was trained on ROP fundus images in combination with vessel segmentation maps obtained from a pre-trained U-net CNN [58. GANs have also been used to synthesize retinal images of diabetic retinopathy, including the ability to control high-level aspects of the presentation [77, 112. While many of the GAN-synthesized images display believable pathologic features, some do contain "checkerboard" and other generation artifacts.

\footnotetext{
${ }^{1}$ Note: this usage of atropine is not approved by the FDA.
} 

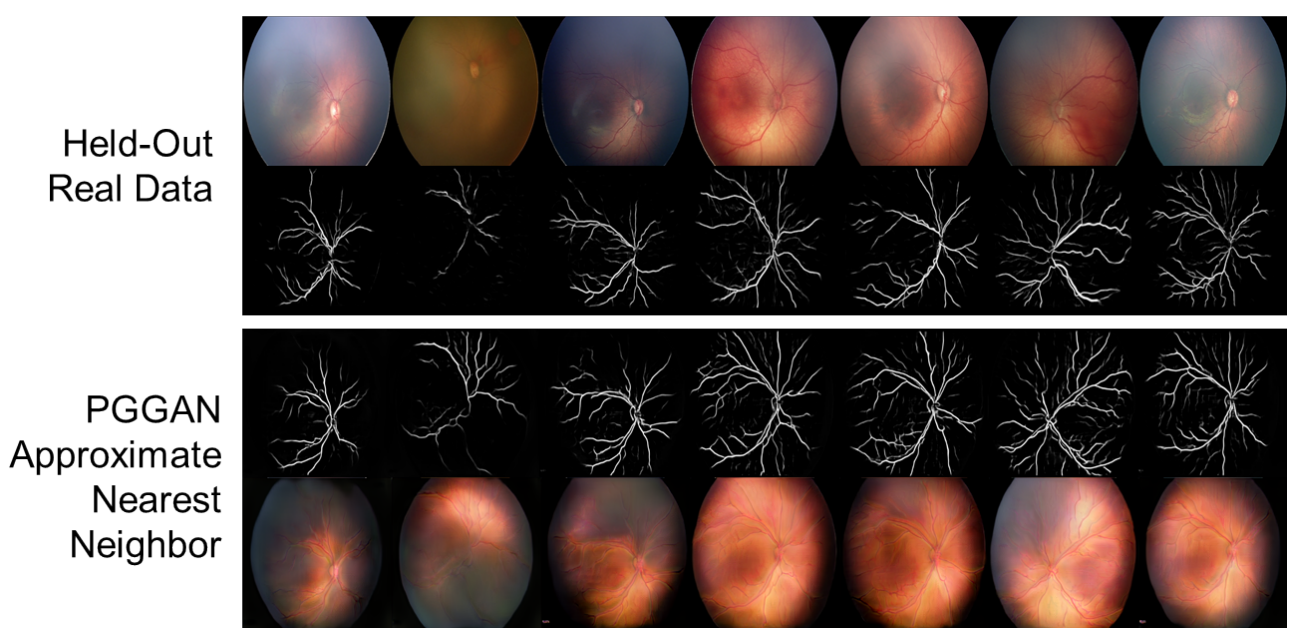

Figure 1. Real (top row) and synthetic (bottom row) fundus images of ROP with their corresponding vessel segmentations [111]. The top row shows real images that were not included in the training set, and the bottom row shows the most similar synthesized images. (Image from $\left[111^{-}\right.$, reused with permission.)

\section{CURRENT LIMITATIONS AND FUTURE DIRECTIONS}

Current applications to pediatric ophthalmology have several limitations that offer avenues for future work.

Disagreement on reference standards An ML classifier's performance is fundamentally limited by the quality of the training data, which are manually labeled by clinicians. However, there is often significant variation of the diagnosis and treatment among physicians, given the same case information 23,42 43, 113, which complicates determination of the correct labels. When ML was used to identify factors influencing ROP experts' decisions for plus disease diagnosis, the most important features were venous tortuosity and vascular branching [23, 43], neither of which are part of the standard "plus disease" definition of arteriolar tortuosity and venular dilatation 114, 115. Most approaches use the majority label from multiple experts as the label for each training instance, or combine the majority label given to imagery with the clinical diagnosis [116. An alternative approach puts cases with any amount of disagreement up for adjudication among the experts, resulting in a consensus label and reducing errors, as demonstrated for diabetic retinopathy 76 .

Need for pediatric-specific models It would be advantageous for pediatric ophthalmology to benefit from the large amount of work in AI for adult ophthalmology. However, due to the unique aspects of pediatric disease manifestation, ML models trained on adult patients may make errors when directly applied to pediatric patients. Transfer learning [56, 57 and multi-task learning 117, 118 techniques may offer a solution to this problem, providing mechanisms to adapt adult models to pediatric patients given a small amount of pediatric ophthalmic data. These methods could also reuse knowledge across models of different diseases or populations - for example, integrating knowledge across multiple smaller pediatric data sets of different ophthalmic diseases to help compensate for the lack of data on any one disease. Notice that, by pretraining on ImageNet, many of the CNN-based methods surveyed here already employ transfer learning of basic image features to compensate for using small data sets; transferring from adult ophthalmic data sets may provide further advantages.

Poor reproducibility and comparability Almost all the ML studies discussed here, even those that focus on the same disease, are trained and evaluated on different data sets. In many cases, the data sets and software source code are not available publicly, complicating reproducibility and scientific comparison across algorithms [59].

Most ML research relies on publicly accessible data sets and software implementations for evaluation and comparison. One simple way to encourage further applications of AI to pediatric ophthalmology is through the public release of data sets in strict compliance with HIPAA regulations, and with special regard to the additional HIPAA restrictions for minors. Even small pediatric ophthalmic data sets could be of use when used in combination with adult data through transfer learning techniques, as mentioned above. For the largest impact, these open data sets should be hosted in a widely used ML repository. 
Lack of temporal information Most of these systems detect disease based upon one snapshot in time, without consideration of longitudinal imaging of the case [16]. In some diseases, such as ROP, rapid change is associated with poorer outcomes [47, 119], suggesting that temporal information may have a role in predicting severe disease.

Uninterpretable "black-box" models Despite their predictive power, the "black-box" nature of most state-of-the-art ML methods, such as deep neural networks, complicates their application in medicine. It is often challenging to quantitatively interpret the inference process of such models, understanding how they arrived at their predictions 120 121. Since they focus on correlations between the input and desired output, in some cases ML models may fixate on confounding factors instead of pathological information 122. Interpretable ML methods provide a potential solution to benefit clinicians, allowing, for example, examination of intermediate decision steps within a deep network, natural language justifications for a decision, or visualization of image features that contribute to a decision 121. While these methods seek to improve the interpretability of black-box models, other approaches seek to improve the predictive power of models that are already interpretable, such as the MediBoost algorithm for growing decision trees via gradient boosting 123 .

\section{CONCLUSION}

There is a large potential for current and future AI applications to pediatric ophthalmology, and there are some diseases, such as NLDO, congenital glaucoma, and congenital ptosis, without any published applications of AI to our knowledge. Automated disease detection, the most common use case, could augment telemedical efforts to broaden access to care, improve efficiency, and result in earlier diagnoses. However, other less-utilized capabilities of this technology, including disease grading and outcome prediction, have the potential to enhance clinical care. All AI methods deployed in clinical care must ultimately match or surpass physician performance while meeting the unique requirements of both clinicians and pediatric patients, suggesting the need to augment evaluations on experimental data sets with clinical trials.

\section{Acknowledgements}

We would like to thank Jing Jin, MD, José Marcio Luna, PhD, and Jorge Mendez for their helpful feedback on this article.

\section{Financial support and sponsorship}

E.E.'s work was partially supported by the Lifelong Learning Machines program from DARPA/MTO under grant \#FA8750-18-2-0117. The funders had no role in the research presented in this article, nor in its preparation, review, or approval. The views and conclusions contained herein are those of the authors and should not be interpreted as necessarily representing the official policies or endorsements, either expressed or implied, of DARPA or the U.S. Government.

\section{Conflicts of interest}

There are no conflicts of interest.

\section{REFERENCES}

Papers of particular interest, published within the annual period of review, have been highlighted as:

- of special interest

- of outstanding interest

1. Gulshan V, Peng L, Coram M, et al. Development and validation of a deep learning algorithm for detection of diabetic retinopathy in retinal fundus photographs. JAMA 2016;316:2402-2410.

2. De Fauw J, Ledsam JR, Romera-Paredes B, et al. Clinically applicable deep learning for diagnosis and referral in retinal disease. Nature Medicine 2018;24:1342-1350.

3. Varadarajan AV, Poplin R, Blumer K, et al. Deep learning for predicting refractive error from retinal fundus images. Investigative Ophthalmology and Visual Science 2018;59:2861-2868.

4. Roach L. Artificial intelligence. Eyenet Magazine 2017:77-83.

5. Consejo A, Melcer T, and Rozema JJ. Introduction to machine learning for ophthalmologists. Seminars in Ophthalmology 2019;34:19-41.

6. Ting DSW, Pasquale LR, Peng L, et al. Artificial intelligence and deep learning in ophthalmology. British Journal of Ophthalmology 2018:2018-313173.

7. Lee A, Taylor P, Kalpathy-Cramer J, and Tufail A. Machine learning has arrived! Ophthalmology 2017;124:1726-1728.

8. Rahimy E. Deep learning applications in ophthalmology. Current Opinion in Ophthalmology 2018;29:254-260. 
9. Caixinha M and Nunes S. Machine learning techniques in clinical vision sciences. Current Eye Research 2017;42:1-15.

10. American Academy of Ophthalmology. The future of artificial intelligence in ophthalmology. AAO Mid-Year Forum 2018.

11. $\mathrm{Du}$ XL, $\mathrm{Li}$ WB, and $\mathrm{Hu}$ BJ. Application of artificial intelligence in ophthalmology. International Journal of Ophthalmology 2018;11:1555-1561.

12. Estes R, Estes D, West C, et al. The American Association for Pediatric Ophthalmology and Strabismus workforce distribution project. Journal of American Association for Pediatric Ophthalmology and Strabismus 2007;11:325-329.

13. Dotan G, Karr DJ, and Levin AV. Pediatric ophthalmology and strabismus fellowship match outcomes, 2000-2015. Journal of American Association for Pediatric Ophthalmology and Strabismus 2017;21:1-181.

14. Gilbert C. Retinopathy of prematurity: A global perspective of the epidemics, population of babies at risk and implications for control. Early Human Development 2008;84:7782.

15. Quinn G. Retinopathy of prematurity blindness worldwide: phenotypes in the third epidemic. Eye and Brain 2016;8:31-36.

16. Worrall DE, Wilson CM, and Brostow GJ. Automated retinopathy of prematurity case detection with convolutional neural networks. In: Workshop on Deep Learning and Data Labeling for Medical Applications (LABELS/DLMIA). 2016:68-76.

17"“. Wang J, Ju R, Chen Y, et al. Automated retinopathy of prematurity screening using deep neural networks. EBioMedicine 2018;35:361-368.

The DeepROP system for ROP detection is trained on the largest data set to date, and is the first to detect severe ROP using fundus images that include the peripheral retina. This deep learning approach demonstrates the potential benefits of fine-grained ROP classification.

18*. Redd TK, Campbell JP, Brown JM, et al. Evaluation of a deep learning image assessment system for detecting severe retinopathy of prematurity. British Journal of Ophthalmology 2018:2018-313156.
The i-ROP-DL deep learning system is the first to detect specific ROP classifications, including clinically significant, type 1 , and type 2 ROP. This model could potentially be a useful telemedical tool for identifying referralwarranted ROP.

19. Rani P, Elagiri Ramalingam R, Rajamani KT, et al. Multiple instance learning: Robust validation on retinopathy of prematurity. International Journal of Control Theory and Applications 2016;9:451-459.

20. Rabinowitz MP, Grunwald JE, Karp KA, et al. Progression to severe retinopathy predicted by retinal vessel diameter between 31 and 34 weeks of postconception age. Archives of Ophthalmology 2007;125:1495-1500.

21. Brown JM, Campbell JP, Beers A, et al. Automated diagnosis of plus disease in retinopathy of prematurity using deep convolutional neural networks. JAMA Ophthalmology 2018;136:803-810.

The i-ROP-DL system detects plus disease in infants with ROP more accurately than the majority of experts in this study. This article highlights a deep learning method with the ability to surpass physician performance.

22. Ataer-Cansizoglu E, Bolon-Canedo V, Campbell JP, et al. Computer-based image analysis for plus disease diagnosis in retinopathy of prematurity: Performance of the "i-ROP" system and image features associated with expert diagnosis. Translational Vision Science \& Technology 2015;4:5.

23. Bolón-Canedoa V, Ataer-Cansizoglub E, Erdogmusb D, et al. Dealing with interexpert variability in retinopathy of prematurity: A machine learning approach. Computer Methods and Programs in Biomedicine 2015;122:1-15.

24. Shah DN, Wilson CM, Ying Gs, et al. Semiautomated digital image analysis of posterior pole vessels in retinopathy of prematurity. Journal of American Association for Pediatric Ophthalmology and Strabismus 2009;13:504506.

25. Wilson CM, Cocker KD, Moseley MJ, et al. Computerized analysis of retinal vessel width and tortuosity in premature infants. Investigative Ophthalmology and Visual Science 2008;49:3577-3585. 
26. Wallace DK, Zhao Z, and Freedman SF. A pilot study using "ROPtool" to quantify plus disease in retinopathy of prematurity. Journal of American Association for Pediatric Ophthalmology and Strabismus 2007;11:381-387.

27. Gelman R, Jiang L, Du YE, et al. Plus disease in retinopathy of prematurity: Pilot study of computer-based and expert diagnosis. Journal of American Association for Pediatric Ophthalmology and Strabismus 2007;11:532540 .

28. Zhang K, Liu X, Jiang J, et al. Prediction of postoperative complications of pediatric cataract patients using data mining. Journal of Translational Medicine 2019;17:2.

29. Jiang J, Liu X, Zhang K, et al. Automatic diagnosis of imbalanced ophthalmic images using a cost-sensitive deep convolutional neural network. BioMedical Engineering OnLine $2017 ; 16: 132$.

30. Long E, Lin H, Liu Z, et al. An artificial intelligence platform for the multihospital collaborative management of congenital cataracts. Nature Biomedical Engineering 2017;1:0024.

31". Lin H, Li R, Liu Z, et al. Diagnostic efficacy and therapeutic decision-making capacity of an artificial intelligence platform for childhood cataracts in eye clinics: A multicentre randomized controlled trial. EClinicalMedicine 2019.

This study describes a multi-center randomized controlled trial evaluating the performance of the CC-Cruiser system for cataract diagnosis and treatment - an important step toward a real-world clinical application of AI to pediatric ophthalmology.

32-. Liu X, Jiang J, Zhang K, et al. Localization and diagnosis framework for pediatric cataracts based on slit-lamp images using deep features of a convolutional neural network. PLOS ONE 2017;12:e0168606.

This study describes a cloud-based ML platform, CC-Cruiser, that accurately detects cataract presence, area, density, and location. Such an approach could detect cataracts in the primary care setting or serve as a complement to the pediatric ophthalmologist's evaluation.

33". Lu J, Fan Z, Zheng C, et al. Automated strabismus detection for telemedicine applications. arXiv 1809.029402018.
This system is the first to detect strabismus remotely from digital facial images. As a telemedical application, this could help determine which children require an ophthalmology referral for strabismus.

34. Chen Z, Fu H, Lo WL, and Chi Z. Strabismus recognition using eye-tracking data and convolutional neural networks. Journal of Healthcare Engineering 2018:7692198.

35. Gramatikov BI. Detecting central fixation by means of artificial neural networks in a pediatric vision screener using retinal birefringence scanning. BioMedical Engineering Online 2017;16:52.

36. Van Eenwyk J, Agah A, Giangiacomo J, and Cibis G. Artificial intelligence techniques for automatic screening of amblyogenic factors. Transactions of the American Ophthalmological Society 2008;106:64-73.

37. Nilsson Benfatto M, Öqvist Seimyr G, Ygge J, et al. Screening for dyslexia using eye tracking during reading. PLOS ONE 2016;11:e0165508.

38. Rello L and Ballesteros M. Detecting readers with dyslexia using machine learning with eye tracking measures. In: Proceedings of the 12th Web for All Conference (W4A). ACM Press, 2015:16.

39. Lin H, Long E, Ding X, et al. Prediction of myopia development among Chinese schoolaged children using refraction data from electronic medical records: A retrospective, multicentre machine learning study. PLOS Medicine 2018;15:e1002674.

This study predicts the development of high myopia in children up to 8 years in advance. Such prediction could potentially be used to guide atropine prophylaxis.

40. Steinkuller PG, Du L, Gilbert C, et al. Childhood blindness. Journal of AAPOS 1999;3:26-32.

41. American Academy of Ophthalmology. Ophthalmologists warn of shortage in specialists who treat premature babies with blinding eye condition. AAO Press Release 2006-07-13 2006.

42. Wallace DK, Quinn GE, Freedman SF, and Chiang MF. Agreement among pediatric ophthalmologists in diagnosing plus and pre-plus disease in retinopathy of prematurity. Journal of AAPOS 2008;12:352-356. 
43. Ataer-Cansizoglu E, Kalpathy-Cramer J, You $\mathrm{S}$, et al. Analysis of underlying causes of interexpert disagreement in retinopathy of prematurity diagnosis. Methods of Information in Medicine 2015;54:93-102.

44. Moral-Pumarega MT, Caserío-Carbonero S, De-La-Cruz-Bértolo J, et al. Pain and stress assessment after retinopathy of prematurity screening examination: Indirect ophthalmoscopy versus digital retinal imaging. BMC Pediatrics 2012;12:132.

45. Gilbert C, Wormald R, Fielder A, et al. Potential for a paradigm change in the detection of retinopathy of prematurity requiring treatment. Archives of Disease in Childhood Fetal and Neonatal Edition 2016:101:F6-F9.

46. Capowski J, Kylstra J, and Freedman S. A numeric index based on spatial frequency for the tortuosity of retinal vessels and its application to plus disease in retinopathy of prematurity. Retina 1995;15:490-500.

47. Heneghan C, Flynn J, O'Keefe M, and Cahill M. Characterization of changes in blood vessel width and tortuosity in retinopathy of prematurity using image analysis. Medical Image Analysis 2002;6:407-429.

48. Swanson C, Cocker KD, Parker KH, et al. Semiautomated computer analysis of vessel growth in preterm infants without and with ROP. British Journal of Ophthalmology 2003;87:1474-1477.

49. Gelman R, Martinez-Perez ME, Vanderveen $\mathrm{DK}$, et al. Diagnosis of plus disease in retinopathy of prematurity using retinal image multiscale analysis. Investigative Opthalmology \& Visual Science 2005;46:4734-4738.

50. Sherry LM, Jin Wang J, Rochtchina E, et al. Reliability of computer-assisted retinal vessel measurement in a population. Clinical and Experimental Ophthalmology 2002;30:179 182.

51. Oloumi F, Rangayyan RM, and Ells AL. Quantification of the changes in the openness of the major temporal arcade in retinal fundus images of preterm infants with plus disease. Investigative Ophthalmology \& Visual Science 2014;55:6728-6735.

52. Lowe DG. Distinctive image features from scale-invariant keypoints. International Journal of Computer Vision 2004;60:91-110.
53. Dietterich TG, Lathrop RH, and LozanoPérez T. Solving the multiple instance problem with axis-parallel rectangles. Artificial Intelligence 2002;89:31-71.

54. Szegedy C, Wei Liu, Yangqing Jia, et al. Going deeper with convolutions. In: IEEE Conference on Computer Vision and Pattern Recognition (CVPR). IEEE, 2015.

55. Ioffe S and Szegedy C. Batch normalization: Accelerating deep network training by reducing internal covariate shift. Proceedings of the International Conference on Machine Learning 2015.

56. Pan SJ and Yang Q. A survey on transfer learning. IEEE Transactions on Knowledge and Data Engineering 2010;22:1345-1359.

57. Weiss K, Khoshgoftaar TM, and Wang D. A survey of transfer learning. Journal of Big Data 2016;3:9.

58. Ronneberger O, Fischer P, and Brox T. Unet: Convolutional networks for biomedical image segmentation. Medical Image Computing and Computer-Assisted Intervention (MICCAI) 2015:234-241.

59. Celi LA, Citi L, Ghassemi M, and Pollard TJ. The PLOS ONE collection on machine learning in health and biomedicine: Towards open code and open data. PLOS ONE 2019;14:e0210232.

60. Early Treatment For Retinopathy Of Prematurity Cooperative Group. Revised indications for the treatment of retinopathy of prematurity: Results of the early treatment for retinopathy of prematurity randomized trial. Arch Ophthalmol 2003;121:1684-1694.

61. Krizhevsky A, Sutskever I, and Hinton GE. ImageNet classification with deep convolutional neural networks. Advances in Neural Information Processing Systems 2012:10971105.

62. Whitman MC and Vanderveen DK. Complications of pediatric cataract surgery. Seminars in Ophthalmology 2014;29:414-420.

63. He K, Zhang X, Ren S, and Sun J. Deep residual learning for image recognition. In: IEEE Conference on Computer Vision and Pattern Recognition (CVPR). IEEE, 2016:770-778.

64. Elston J. Concomitant strabismus. In: Paediatric Ophthalmology. Ed. by Taylor D. Oxford: Blackwell Science, 1997. 
65. Adams GGW and Sloper JJ. Update on squint and amblyopia. Journal of the Royal Society of Medicine 2003;96:3-6.

66. Mojon-Azzi SM and Mojon DS. Strabismus and employment: The opinion of headhunters. Acta Ophthalmologica 2009;87:784788.

67. Mojon-Azzi SM, Kunz A, and Mojon DS. The perception of strabismus by children and adults. Graefe's Archive for Clinical and Experimental Ophthalmology 2011;249:753757.

68. Mohney BG, McKenzie JA, Capo JA, et al. Mental illness in young adults who had strabismus as children. Pediatrics 2008;122:10331038.

69. American Academy of Pediatrics. Visual system assessment in infants, children, and young adults by pediatricians. Pediatrics 2016;137:e20153596.

70. Quinlan J. C4.5: Programs for Machine Learning. Morgan Kaufmann Publishers, 1993.

71. Ikuno Y. Overview of the complications of high myopia. Retina 2017;37:2347-2351.

72. Clark TY and Clark RA. Atropine 0.01\% eyedrops significantly reduce the progression of childhood myopia. Journal of Ocular Pharmacology and Therapeutics 2015;31:541-545.

73. Chia A, Lu QS, and Tan D. Five-year clinical trial on atropine for the treatment of myopia 2: Myopia control with atropine $0.01 \%$ eyedrops. Ophthalmology 2016;123:391-399.

74. Gargeya R and Leng T. Automated identification of diabetic retinopathy using deep learning. Ophthalmology 2017;124:962-969.

75. Soto-Pedre E, Navea A, Millan S, et al. Evaluation of automated image analysis software for the detection of diabetic retinopathy to reduce the ophthalmologists' workload. Acta Ophthalmologica 2014.

76. Krause J, Gulshan V, Rahimy E, et al. Grader variability and the importance of reference standards for evaluating machine learning models for diabetic retinopathy. Ophthalmology 2018;125:1264-1272.

77. Pujitha AK and Sivaswamy J. Retinal image synthesis for CAD development. Proceedings of the International Conference on Image Analysis and Recognition 2018:613-621.
78. Lee CS, Baughman DM, and Lee AY. Deep learning is effective for classifying normal versus age-related macular degeneration OCT images. Ophthalmology Retina 2017;1:322327.

79. Rohm M, Tresp V, Müller M, et al. Predicting visual acuity by using machine learning in patients treated for neovascular agerelated macular degeneration. Ophthalmology 2018;125:1028-1036.

80. Klimscha S, Waldstein SM, Schlegl T, et al. Spatial correspondence between intraretinal fluid, subretinal fluid, and pigment epithelial detachment in neovascular age-related macular degeneration. Investigative Opthalmology \& Visual Science 2017;58:4039.

81. Bogunovic H, Montuoro A, Baratsits M, et al. Machine learning of the progression of intermediate age-related macular degeneration based on OCT imaging. Investigative Opthalmology \& Visual Science 2017;58:BIO141.

82. Grassmann F, Mengelkamp J, Brandl C, et al. A deep learning algorithm for prediction of age-related eye disease study severity scale for age-related macular degeneration from color fundus photography. Ophthalmology 2018;125:1410-1420.

83. Schlanitz FG, Baumann B, Kundi M, et al. Drusen volume development over time and its relevance to the course of age-related macular degeneration. British Journal of Ophthalmology 2017.

84. Ohsugi H, Tabuchi H, Enno H, and Ishitobi $\mathrm{N}$. Accuracy of deep learning, a machinelearning technology, using ultra-wide-field fundus ophthalmoscopy for detecting rhegmatogenous retinal detachment. Scientific Reports 2017;7:9425.

85. Zhen Y, Chen H, Zhang X, et al. Assessment of central serous chorioretinopathy (CSC) depicted on color fundus photographs using deep learning. arXiv 1901.04540 2019.

86. Schlegl T, Waldstein SM, Bogunovic H, et al. Fully automated detection and quantification of macular fluid in OCT using deep learning. Ophthalmology 2018;125:549-558.

87. Prahs P, Radeck V, Mayer C, et al. OCTbased deep learning algorithm for the evaluation of treatment indication with antivascular endothelial growth factor medications. Graefe's Archive for Clinical and Experimental Ophthalmology 2017;256:91-98. 
88. Bagheri A, Persano Adorno D, Rizzo P, et al. Empirical mode decomposition and neural network for the classification of electroretinographic data. Medical \& Biological Engineering \& Computing 2014;52:619-628.

89. Kermany DS, Goldbaum M, Cai W, et al. Identifying medical diagnoses and treatable diseases by image-based deep learning. Cell 2018;172:1122-1131.

90. Omodaka K, An G, Tsuda S, et al. Classification of optic disc shape in glaucoma using machine learning based on quantified ocular parameters. PLOS ONE 2017;12:e0190012.

91. Li Z, He Y, Keel S, et al. Efficacy of a deep learning system for detecting glaucomatous optic neuropathy based on color fundus photographs. Ophthalmology 2018;125:11991206.

92. Martin KR, Mansouri K, Weinreb RN, et al. Use of machine learning on contact lens sensor-derived parameters for the diagnosis of primary open-angle glaucoma. American Journal of Ophthalmology 2018;194:46-53.

93. Clarke GP and Burmeister J. Comparison of intraocular lens computations using a neural network versus the Holladay formula. Journal of Cataract \& Refractive Surgery 1997;23:1585-1589.

94. Hwang ES, Perez-Straziota CE, Kim SW, et al. Distinguishing highly asymmetric keratoconus eyes using combined Scheimpflug and spectral-domain OCT analysis. Ophthalmology 2018;125:1862-1871.

95. Edwards TL, Xue K, Meenink HC, et al. First-in-human study of the safety and viability of intraocular robotic surgery. Nature Biomedical Engineering 2018;2:649-656.

96. Lahiri A, Roy AG, Sheet D, and Biswas PK. Deep neural ensemble for retinal vessel segmentation in fundus images towards achieving label-free angiography. International Conference of the IEEE Engineering in Medicine and Biology Society (EMBC) 2016:13401343.

97. Maji D, Santara A, Ghosh S, et al. Deep neural network and random forest hybrid architecture for learning to detect retinal vessels in fundus images. International Conference of the IEEE Engineering in Medicine and Biology Society (EMBC) 2015:3029-3032.
98. Knudtson MD, Lee KE, Hubbard LD, et al. Revised formulas for summarizing retinal vessel diameters. Current Eye Research 2003;27:143-149.

99. Ng J, Clay ST, Barman SA, et al. Maximum likelihood estimation of vessel parameters from scale space analysis. Image and $\mathrm{Vi}$ sion Computing 2010;28:55-63.

100. Poplin R, Varadarajan AV, Blumer K, et al. Prediction of cardiovascular risk factors from retinal fundus photographs via deep learning. Nature Biomedical Engineering 2018;2:158164.

101. Lewis TL, Mondloch CJ, Maurer D, et al. The effect of early visual deprivation on the development of face detection. Developmental Science 2013;16:728-742.

102. Grady CL, Mondloch CJ, Lewis TL, and Maurer D. Early visual deprivation from congenital cataracts disrupts activity and functional connectivity in the face network. Neuropsychologia 2014;57:122-139.

103". Vogelsang L, Gilad-Gutnicka S, Ehrenberga E, et al. Potential downside of high initial visual acuity. Proceedings of the National Academy of Sciences 2018;115:11333-11338.

This article proposes that high initial acuity can disrupt visual development, and suggests it as an explanation of why adults with a history of congenital cataract surgery in infancy may exhibit deficient facial recognition. Their hypothesis is supported by experimental results that use convolutional neural networks to model visual development, and could be used to improve neural network training.

104. Fraz MM, Rudnicka AR, Owen CG, and Barman SA. Delineation of blood vessels in pediatric retinal images using decision trees-based ensemble classification. International Journal of Computer Assisted Radiology and Surgery 2014;9:795-811.

105. Owen CG, Rudnicka AR, Mullen R, et al. Measuring retinal vessel tortuosity in 10-yearold children: Validation of the ComputerAssisted Image Analysis of the Retina (CAIAR) program. Investigative Opthalmology \& Visual Science 2009;50:2004-2010.

106. Goodfellow I, Pouget-Abadie J, Mirza M, et al. Generative adversarial nets. Advances in Neural Information Processing Systems 2014;27:2672-2680. 
107. Zhao H, Li H, and Cheng L. Synthesizing filamentary structured images with GANs. arXiv 1706.021852017.

108. Costa P, Galdran A, Meyer MI, et al. End-to-end adversarial retinal image synthesis. IEEE Transactions on Medical Imaging 2018;37:781-791.

109. Yi X, Walia E, and Babyn P. Generative adversarial network in medical imaging: A review. arXiv 1809.072942019.

110. Finlayson SG, Kohane IS, and OakdenRayner L. Towards generative adversarial networks as a new paradigm for radiology education. arXiv:1812.01547 2018.

111. Beers A, Brown J, Chang K, et al. Highresolution medical image synthesis using progressively grown generative adversarial networks. arXiv 1805.03144 2018.

This is the first example of realistic synthesized ROP fundoscopic images. Synthesized images would be an effective way to augment data sets and resident education without compromising patient privacy.

112. Niu Y, Gu L, Lu F, et al. Pathological evidence exploration in deep retinal image diagnosis. Proceedings of the AAAI Conference on Artificial Intelligence 2019.

113. Chiang MF, Jiang L, Gelman R, et al. Interexpert agreement of plus disease diagnosis in retinopathy of prematurity. Archives of Ophthalmology 2007;125:875-880.

114. Committee for the Classification of Retinopathy of Prematurity. An international classification of retinopathy of prematurity. Archives of Ophthalmology 1984;102:1130 1134.

115. International Committee for the Classification of Retinopathy of Prematurity. The International Classification of Retinopathy of Prematurity revisited. Archives of Ophthalmology 2005;123:991-999.

116. Ryan MC, Ostmo S, Jonas K, et al. Development and evaluation of reference standards for image-based telemedicine diagnosis and clinical research studies in ophthalmology. AMIA Annual Symposium Proceedings 2014:1902-1910.

117. Ruder S. An overview of multi-task learning in deep neural networks. arXiv 1706.05098 2017.
118. Zhang $\mathrm{Y}$ and Yang Q. An overview of multi-task learning. National Science Review 2018;5:30-43.

119. Wallace DK, Kylstra JA, and Chesnutt DA. Prognostic significance of vascular dilation and tortuosity insufficient for plus disease in retinopathy of prematurity. Journal of AAPOS 2000;4:224-229.

120. Doshi-Velez F and Kim B. Towards a rigorous science of interpretable machine learning. arXiv:1702.08608 2017.

121. Gilpin LH, Bau D, Yuan BZ, et al. Explaining explanations: An overview of interpretability of machine learning. Proceedings of the 5th IEEE International Conference on Data Science and Advanced Analytics (DSAA) 2018.

122. Zech JR, Badgeley MA, Liu M, et al. Variable generalization performance of a deep learning model to detect pneumonia in chest radiographs: A cross-sectional study. PLOS Medicine 2018;15:e1002683.

123. Valdes G, Luna JM, Eaton E, et al. MediBoost: A patient stratification tool for interpretable decision making in the era of precision medicine. Scientific Reports 2016;6:37854. 


\section{ONLINE SUPPLEMENT: A BRIEF OVERVIEW OF AI AND ML}

Artificial intelligence (AI) is the broad field concerned with the study of intelligence and its computational manifestation within machines. It spans a broad set of problems that are all interrelated, from basic search (e.g., route finding on a map, or sequences of moves in a chess game) to logical reasoning (e.g., theorem proving, logistics planning) to reasoning under uncertainty (e.g., Bayesian abductive reasoning) to multi-agent systems (e.g., markets of trading agents) to robotics (e.g., computer vision and perception, control of dynamical systems) to learning.

Machine learning (ML) is perhaps the largest subfield of AI and is concerned with this latter problem of learning from experience (i.e., data). Most recent news headlines and research concerning the application of AI techniques to problems in other disciplines (including the title of this article) would more precisely be termed applications of ML. Modern statistical ML is primarily concerned with the optimization of a model (e.g., a classification or regression model) to fit a given set of training data in such a manner that the model will be able to generalize to new data.

As a simple example, the training data might consist of demographical, biometric, and imaging data of a chosen cohort of 10,000 patients gathered from hospital records. Each record (called a data instance) could be characterized as a set of categorical, ordinal, and numeric features that are derived from the patient's record, and would be labeled according to the patient's diagnosis. ML algorithms could then train a classifier model (e.g., a decision tree, logistic regression) to predict the labeled diagnosis of a patient given the set of features derived from their record. Critically, the performance of the classifier should be assessed on new patients from the same population (i.e., patients with similar demographics that were not present in the training data), using application-dependent metrics (such as accuracy, sensitivity/specificity, receiver operating characteristic (ROC) curves).

This example focused on a supervised learning setting, in which each patient's data instance had a corresponding categorical label and we trained a classification model. If the labels had instead been numeric values, we could have trained a regression model using other supervised learning algorithms. Other settings include semi-supervised learning, in which only some data instances are labeled; unsupervised learning, which focuses on discovering patterns in unlabeled data (e.g, clusters of patients with similar biomarkers), and reinforcement learning, which seeks to learn a policy that can determine sequences of actions to execute to achieve a goal (e.g., the sequence of treatments to administer to an ICU patient, or the movements a robot should perform to tie a ligature). There are numerous different ML techniques, which vary according to the model representation (e.g., decision trees, linear classifiers, neural networks, logical rules), the mathematical technique used to optimize the model (e.g., greedy heuristics, gradient descent, evolutionary computation), and the evaluation metric used to assess the quality of model fit to the data (e.g., accuracy, precision and recall, posterior probability). Note that these metrics focus on performance on data and do not necessarily relate to the model acquiring generalizable knowledge. Consequently, ML models are learning patterns of correlations between the inputs and the desired outputs, not causal knowledge. This may cause them to exploit confounding details instead of physiological aspects. For example, an image classifier tasked with predicting disease severity might erroneously focus on identifying the type of camera (portable vs. fixed) or the presence of chest tubes or other medical devices, rather than pathological information, simply because these other confounding details are highly correlated with the desired output 122 .

Deep learning (DL) methods are one subgroup of ML techniques that have shown exceptional impact to a wide variety of applications. Although DL techniques have been studied for decades, recent advances in computational algorithms and hardware have enabled these models to be trained at scale on large data sets, leading to their impact. DL is concerned with training models with numerous layers of processing, such as deep neural networks. Convolutional neural networks (CNNs) are one popular type of deep network that are often used for image classification. These models take raw input, such as a fundus photograph, and extract layered features from the input image, where higher levels of the deep neural network typically focus on increasingly abstract features that are built upon lower-level features. This automatic discovery of features is called representation learning, since the model identifies commonalities within the given input data as a way to re-represent it at different levels of abstraction. Although fundamentally an unsupervised learning technique, deep learning models can easily be adapted for classification, regression, and reinforcement learning. Despite its success and popularity, DL typically requires large data sets for training (e.g., thousands or hundreds of thousands or millions of examples, depending on the complexity of the decision), which may be problematic in certain medical applications. 